\title{
The characteristics and outcomes of critically III patients with COVID-19 who received systemic thrombolysis for presumed pulmonary embolism: an observational study
}

\author{
Matsuo So ${ }^{1} \cdot$ David J. Steiger $^{2} \cdot$ Mai Takahashi $^{1} \cdot$ Natalia N. Egorova $^{3} \cdot$ Toshiki Kuno $^{1}$ \\ Accepted: 3 May 2021 / Published online: 8 May 2021 \\ (c) The Author(s), under exclusive licence to Springer Science+Business Media, LLC, part of Springer Nature 2021
}

\begin{abstract}
Coronavirus disease 2019 (COVID-19) is associated with abnormal hemostasis, autopsy evidence of systemic microthrombosis, and a high prevalence of venous thromboembolic disease. Tissue plasminogen activator (tPA) has been used in patients with critically ill COVID-19 with high clinical suspicion of pulmonary embolism (PE). A retrospective cohort study of 6095 hospitalized COVID-19 patients at 5 acute care hospitals in New York was conducted. 57 patients received tPA for presumed PE during March 10th to April 27th. The mean age was $60.8 \pm 10.8$ years, and 71.9\% (41/57) were male. We defined strongly suspected PE among 75.4\% (43/57) of patients who had acute worsening of hypoxia and acute hypotension requiring pressors. The findings suggestive of PE included right ventricular (RV) strain in 15.8\% (9/57), deep venous thrombosis (DVT) in 7.0\% (4/57), increased dead space ventilation ( $\mathrm{Vd}$ ) in 31.6\% (18/57) of patients, respectively. RV strain and RV thrombus were present in 3.5\% (2/57), RV strain and DVT in 5.3\% (3/57), RV strain and increased Vd in 8.8\% (5/57), and DVT and increased Vd in 3.5\% (2/57) of patients. Chest CT Angiography was not performed in any of the patients. Following tPA infusion, $49.1 \%$ (28/57) of patients demonstrated improvement. Six patients (10.5\%) survived to discharge, of whom 2 received extracorporeal membrane oxygenation and were transferred to other facilities for lung transplant, 2 were discharged home, and 2 were discharged to a rehabilitation facility. However, overall mortality was $89.5 \%$. The utility of tPA for critically ill patients with COVID-19 and presumed PE warrants further studies.
\end{abstract}

Keywords COVID-19 $\cdot$ Tissue plasminogen activator $\cdot$ Respiratory insufficiency $\cdot$ Pulmonary embolism $\cdot$ Shock

\section{Highlights}

- Coronavirus disease 2019 (COVID-19) has been reported to be associated with abnormal hemostasis and a high prevalence of venous thromboembolic disease.

Toshiki Kuno

toshiki.kuno@mountsinai.org

1 Department of Medicine, Icahn School of Medicine at Mount Sinai, Mount Sinai Beth Israel, First Avenue, 16th Street, New York City, NY 10003, USA

2 Department of Pulmonary, Critical Care and Sleep Medicine, Icahn School of Medicine at Mount Sinai, Mount Sinai Beth Israel, New York City, NY, USA

3 Department of Population Health Science and Policy, Icahn School of Medicine at Mount Sinai, New York City, NY, USA
- Tissue plasminogen activator (tPA) has been generally used in clinically unstable patients with presumed PE.

- A few case series of successful use of tPA in critically-ill hypoxic or hypotensive COVID-19 patients have been reported.

- The use of tPA for critically ill unstable COVID-19 patients showed improvement in clinical parameters in $49.1 \%$ in our study cohort.

- Overall mortality of patients who received tPA were $89.5 \%$. The utility of tPA for critically ill unstable COVID-19 patients warrants further studies.

\section{Introduction}

Coronavirus disease 2019 (COVID-19) is caused by a novel coronavirus, severe acute respiratory syndrome coronavirus 2 (SARS-CoV-2) [1], which is known to cause severe pneumonia and critical illness leading to multiple organ damage. 
COVID-19 can induce a hypercoagulable state associated with elevated D-dimer levels, venous thromboembolism (VTE), disseminated intravascular coagulation (DIC) and bleeding [2]. The mechanisms involved are multiple and include endothelial injury, as evidenced by elevated von Willebrand factor and Factor VIII levels [3]. Increase in proinflammatory cytokines secondary to COVID-19 may play a direct role in creating a prothrombotic state [4]. Autopsy studies of COVID-19 patients have demonstrated fibrin thrombi in small vessels and capillaries [5]. Multiple case series of intensive care unit (ICU) patients have reported high rates of VTE despite prophylactic doses of anticoagulation $[6,7]$. There has been a debate in the literature with regard to recommending the empirical use of therapeutic doses of anticoagulation, using unfractionated heparin (UFH) or low molecular weight heparin (LMWH) in critically ill COVID-19 patients who do not have a firm diagnosis of a deep venous thrombosis (DVT) or PE but have evidence of sudden respiratory decompensation, or right heart strain on echocardiogram suggestive of a possible PE. [8,9]. The specific use of UFH and LMWH has been advocated because of the possible beneficial anti-inflammatory effects of these anticoagulation therapies [9]. Tissue plasminogen activator (tPA) has been administered to patients with COVID19 and a suspected PE. In one case series, tPA to patients with increasingly severe hypoxia on the verge of intubation, too unstable for chest computed tomography angiography (CTA), was associated with a favorable response. In this study, there was neither evidence of right ventricular (RV) dilatation, RV hypokinesia by echocardiography nor DVT diagnosed [10]. In a second case series, tPA was given to patients with presumed PE who were ventilated and had physiological evidence of increased dead space ventilation (Vd) manifest as an increased arterial partial carbon dioxide pressure $[11,12]$. The aim of our study was to describe the clinical characteristics and outcomes of critically ill COVID19 patients who received tPA treatment for a presumed PE.

\section{Materials and methods}

We obtained de-identified data from the Mount Sinai Data Warehouse (MSDW) an institutional database that contains all information related to health encounters at five Mount Sinai facilities. From 6095 hospitalized patients admitted between March 10th and April 27th 2020 with reverse transcriptase polymerase-chain-reaction (RT-PCR) confirmed SARS-CoV-2 infection by nasopharyngeal or oropharyngeal swab, we performed a retrospective analysis of data from patients who received tPA during hospitalization. The Institutional Review Board of Icahn School of Medicine at Mount Sinai approved the research project (IRB \#2000495) and the research was conducted in accordance with the principles of the Declaration of Helsinki. The waiver of patients' informed consent was also approved by the institutional review boards.

We assessed patient's demographic and clinical characteristics, treatment for COVID-19 including details of the management of acute respiratory distress syndrome (ARDS), anticoagulation therapies prior to tPA infusion, and serum D-dimer values at the time of tPA administration. We evaluated evidence supporting a diagnosis of PE, the indications for tPA infusion, complications from tPA, and mortality outcomes. Anticoagulation therapies prior to the administration of tPA included therapeutic anticoagulation were the patient received either LMWH (enoxaparin $1 \mathrm{mg}$ / $\mathrm{kg}$ every $12 \mathrm{~h}$ or $1.5 \mathrm{mg} / \mathrm{kg}$ every $24 \mathrm{~h}$ ), therapeutic UFH by continuous infusion or full dose direct oral anticoagulants (DOAC). Prophylactic anticoagulation administered prior to tPA included subcutaneous UFH injection 5000 units every $12 \mathrm{~h}$, or LMWH (enoxaparin $40 \mathrm{mg}$, every $24 \mathrm{~h}$ ). Patients were evaluated for a possible PE when patients had worsening hypoxia, there was development of acute hypotension, if hypoxia developed despite maximal ventilatory support, or hypotension was refractory to vasopressor therapy. A presumptive diagnosis of high-risk PE was made based on pulmonary embolism response team (PERT) consortium guideline [13] if there was echo evidence of right atrial or RV thrombus, RV strain, venous duplex evidence of a DVT [14], and increased Vd. The presence of RV strain was defined by RV dilation (right ventricle/left ventricle ratio $>1: 1$ ), septal motion abnormality and RV dysfunction [15]. The presence of hypercapnia despite adequate minute ventilation was deemed to be suggestive of pulmonary embolism due to extensive increased $\mathrm{Vd}$ [16]. The quantitative assessment of increased dead space ventilation by calculating the fraction of dead space ventilation $(\mathrm{Vd} / \mathrm{Vt})$ was not performed per chart review. Major bleeding complications were defined as fatal bleeding, symptomatic bleeding in a critical area or organ, such as intracranial, intraspinal, intraocular, retroperitoneal, intra-articular or pericardial, or intramuscular with compartment syndrome, or bleeding causing a fall in hemoglobin level of $2 \mathrm{~g} / \mathrm{dl}$ or more, or leading to transfusion of two or more units of whole blood or red cells. [17] Absolute contraindications to tPA for PE included prior history of intracranial hemorrhage, a cerebral vascular lesion, or malignant intracranial neoplasm, ischemic stroke within preceding 3 months, suspected aortic dissection, active bleeding or bleeding diathesis and significant closed-head trauma or facial trauma within prior 3 months.

\section{Statistical analysis}

Continuous variables are presented as mean \pm standard deviation or median [interquartile range] depending on what is appropriate for the data distribution, and categorical 
variables were expressed as percentages. All statistical calculations and analyses were performed on $\mathrm{R}$ (version 3.6.2, R Foundation for Statistical Computing, Vienna, Austria).

\section{Results}

A total of 64 patients received tPA during hospitalization. Five patients were given tPA for ischemic stroke and 2 patients for clearance of catheter occlusion, these 7 patients were excluded from the study. Among the 57 patients who were eligible ( $0.94 \%$ out of 6095 hospitalized patients with COVID-19), the mean age was $60.8 \pm 10.8$ years, $71.9 \%$ (41/57) of patients were male. The most common comorbidity was hypertension $56.1 \%$ (32/57), followed by obesity $54.4 \%$ (31/57), (mean body mass index $31.5 \pm 7.0 \mathrm{~kg} / \mathrm{m}^{2}$ ), diabetes mellitus $40.4 \%$ (23/57). With respect to past history of respiratory diseases, asthma was reported in $12.3 \%$ of patients (7/57), chronic obstructive pulmonary disease 5.3\% (3/57), interstitial lung disease 5.3\% (3/57) and obstructive sleep apnea $8.8 \%(5 / 57)$, and $7.0 \%$ (4/57) of patients were current smokers. No patients had a contraindication for tPA administration (Table 1). Pharmacological treatment for COVID-19 included 77.2\% (44/57) received corticosteroids, $26.3 \%$ (15/57) were treated with tocilizumab. Therapeutic doses of anticoagulation were given to $73.4 \%$ (42/57) of patients and prophylactic doses of chemical prophylaxis were administered to $22.8 \%$ (13/57) of patients. The management of ARDS included prone positioning in 57.9\% (33/57) of patients, and $77.2 \%$ (44/57) received neuromuscular blockade. Trials of pulmonary vasodilator therapy (nitric oxide inhalation or epoprostenol inhalation) were reported in $35.1 \%$ (20/57) of patients. 7\% (4/57) of patients received extracorporeal membrane oxygenation (ECMO) for respiratory and circulatory support (Table 2 ).

98.2\% (56/57) of patients received invasive ventilation and 1 patient was on non-invasive positive pressure ventilation (NIPPV). Full dose tPA was given to patients with the following single abnormalities: echo evidence of RV strain in $15.8 \%$ patients (9/57), DVT in $7.0 \%$ (4/57), increased Vd in $31.6 \%(18 / 57)$ of patients. Patients who received tPA had 2 abnormal findings that included RV strain and RV thrombus in 3.5\% (2/57), RV strain and DVT in 5.3\% (3/57), RV strain and increased Vd in 8.8\% (5/57), DVT and increased $\mathrm{Vd}$ in $3.5 \%$ (2/57). $24.6 \%$ (14/57) of patients received tPA for suspected PE in the clinical context of worsening hypoxia and hypotension without information of echocardiographic evidence of RV strain, RV thrombus, DVT, or increased $\mathrm{Vd}$. The mean serum level of D-dimer before tPA was 10.5 [3.4-19.2] $\mu \mathrm{g} / \mathrm{ml}$ (Table 3).

Chest CTA was not performed in any of the study patients due to the patients' clinical instability secondary to critical illness. After tPA infusion, 49.1\% (28/57) of patients showed
Table 1 Baseline characteristics of COVID-19 patients who received tissue plasminogen activator

\begin{tabular}{ll}
\hline Patient Characteristics & $\begin{array}{l}\text { Total number of } \\
\text { patients }(\mathrm{n}=57)\end{array}$ \\
\hline Age (yr), mean \pm SD & $60.8 \pm 10.8$ \\
Sex, $\mathrm{n}(\%)$ & \\
Male & $41(71.9)$ \\
Female & $16(28.1)$ \\
Comorbidities, $\mathrm{n}(\%)$ & \\
Asthma & $7(12.3)$ \\
Chronic obstructive lung disease & $3(5.3)$ \\
Interstitial lung disease & $3(5.3)$ \\
Smoking history & \\
Current smoker & $4(7.0)$ \\
Former smoker & $15(26.3)$ \\
Never smoker & $26(45.6)$ \\
Hypertension & $32(56.1)$ \\
Obstructive sleep apnea & $5(8.8)$ \\
Obesity & $31(54.4)$ \\
Body mass index (kg/m $\left.{ }^{2}\right)$, mean \pm SD & $31.5 \pm 7.0$ \\
Diabetes mellitus & $23(40.4)$ \\
Hyperlipidemia & $16(28.1)$ \\
Coronary artery disease & $9(15.8)$ \\
Chronic kidney disease & $1(1.8)$ \\
Human immunodeficiency virus infection & $1(1.8)$ \\
Malignancy & $2(3.5)$ \\
Multiple myeloma & $1(1.8)$ \\
Chronic lymphocytic leukemia & $1(1.8)$ \\
Hypothyroidism & $3(5.3)$ \\
Atrial fibrillation & $1(1.8)$ \\
Heart failure with reduced ejection fraction & $2(3.5)$ \\
Heart failure with preserved ejection fraction & $2(3.5)$ \\
Non-alcoholic fatty liver disease & $1(1.8)$ \\
Rheumatoid arthritis & $2(3.5)$ \\
Parkinson's disease & $1(1.8)$ \\
Hospital LOS (d), median [IQR] & $17[11,27.5]$ \\
\hline & \\
\hline &
\end{tabular}

Value is shown as mean $\pm \mathrm{SD}$ or median [Q1, Q3]

$S D$ standard deviation; $L O S$ length of stay

initial improvement in either of $\mathrm{PaO}_{2} / \mathrm{FiO}_{2}$ ratio, blood pressure or partial arterial pressure of carbon dioxide $\left(\mathrm{PaCO}_{2}\right)$, which led to successful weaning of ventilator settings or vasopressors. The duration of ventilator dependence following tPA was $5[1,11.5]$ days. Mild hemoptysis was reported in 1 patient after tPA. This patient had previously required suctioning of blood from the endotracheal tube 1 day before tPA infusion, while receiving UFH continuous infusion. The hemoglobin level before tPA was $7.8 \mathrm{~g} / \mathrm{dl}$ which dropped to $6.6 \mathrm{~g} / \mathrm{dl} 2$ days after tPA and required 1 unit of red blood cell transfusion. Discontinuation of tPA was not required for any of the patients (Table 4). 
Table 2 Treatment for patients with COVID-19 related ARDS

\begin{tabular}{ll}
\hline COVID-19 treatment, $\mathrm{n}(\%)$ & $\begin{array}{l}\text { Total number of } \\
\text { patients }(\mathrm{n}=57)\end{array}$ \\
\hline Azithromycin & $48(84.2)$ \\
Hydroxychloroquine & $50(87.7)$ \\
Remdesivir & $3(5.3)$ \\
Tocilizumab & $15(26.3)$ \\
Sarilumab & $2(3.5)$ \\
Anakinra & $1(1.8)$ \\
Corticosteroids & $44(77.2)$ \\
Convalescent plasma & $7(12.3)$ \\
Mesenchymal stem cell therapy & $3(5.3)$ \\
Anticoagulation therapies, $\mathrm{n}(\%)$ & \\
Therapeutic anticoagulation & $42(73.4)$ \\
LMWH & $25(43.9)$ \\
UFH & $16(28.1)$ \\
DOAC & $1(1.8)$ \\
Preventive anticoagulation & $13(22.8)$ \\
LMWH & $6(10.5)$ \\
UFH & $7(12.3)$ \\
No anticoagulation & $2(3.5)$ \\
ARDS treatment, $\mathrm{n}(\%)$ & \\
Prone positioning & $33(57.9)$ \\
Neuromuscular blockade & $44(77.2)$ \\
Pulmonary vasodilator & $20(35.1)$ \\
Organ support, $\mathrm{n}(\%)$ & \\
Invasive Ventilation & $57(100)$ \\
Tracheostomy & $12(21.1)$ \\
Hemodialysis & $15(26.3)$ \\
ECMO & $4(7.0)$ \\
\hline
\end{tabular}

LMWH low molecular weight heparin; $U F H$ unfractionated heparin; $D O A C$ direct oral anticoagulant; $A R D S$ acute respiratory distress syndrome; $E C M O$ extracorporeal membrane oxygenation

The overall mortality rate was $89.5 \%$ (51/57). Among patients who had improvement after tPA infusion, $7.0 \%$ (4/57) of patients survived and $42.1 \%$ (24/57) of patients died, and among patients who had no improvement after tPA infusion, 3.5\% (2/57) of patients survived and 47.4\% (27/57) of patients eventually died (Table 4$)$. The mortality of patients with presumed PE based on clinical, echocardiographic or physiological findings suggestive of PE was $86.0 \%$ (37/43) and the mortality of patients who received tPA on the assumption that they have PE based on the rapid clinical deterioration even without clinical echocardiographic or physiological findings suggestive of PE was $100 \%(14 / 14)$. Of the 6 patients who survived their hospital course, two patients required ECMO and were transferred to an outside hospital for lung transplant evaluation. Two patients were discharged home and two patients were
Table 3 Tissue plasminogen activator indications for patients with presumptive diagnosis of pulmonary embolism in deteriorating hypoxia and shock and types of respiratory support when receiving tPA

\begin{tabular}{ll}
\hline tPA indications, $\mathrm{n}(\%)$ & $\begin{array}{l}\text { Total number of } \\
\text { patients }(\mathrm{n}=57)\end{array}$ \\
\hline Sole RV strain & $9(21.1 \%)$ \\
RV strain and RV thrombus & $2(3.5 \%)$ \\
RV strain and DVT & $3(5.3 \%)$ \\
RV strain and increased Vd & $5(8.8 \%)$ \\
Sole DVT on US & $4(7.0 \%)$ \\
Sole Increased Vd & $18(31.6 \%)$ \\
DVT and increased Vd & $2(3.5 \%)$ \\
Hypoxia and shock without clear evidence of PE & $14(24.6 \%)$ \\
Respiratory support at tPA, n (\%) & \\
Non-rebreather mask & $0(0 \%)$ \\
High flow nasal cannula & $0(0 \%)$ \\
Bilevel positive airway ventilation & $1(1.8 \%)$ \\
Invasive mechanical ventilator & $56(98.2 \%)$ \\
D-dimer ( $\mu \mathrm{g} / \mathrm{ml})$, median [IQR] & $10.5[3.4-19.2]$ \\
\hline
\end{tabular}

$t P A$ tissue plasminogen activator; $R V$ right ventricle; FOCUS focused cardiac ultrasound; $D V T$ deep venous thrombosis; $U S$ ultrasound; $P E$ pulmonary embolism

Table 4 Improvement with successful weaning of ventilator settings and vasopressors, outcomes and complications

\begin{tabular}{ll}
\hline Outcomes and complications, $\mathrm{n}(\%)$ & $\begin{array}{l}\text { Total number of } \\
\text { patients }(\mathrm{n}=57)\end{array}$ \\
\hline Death & $51(89.5 \%)$ \\
Improvement after tPA and survived & $4(7.0 \%)$ \\
Improvement after tPA and died & $24(42.1 \%)$ \\
No improvement after tPA and survived & $2(3.5 \%)$ \\
No improvement after tPA and died & $27(47.4 \%)$ \\
Major bleeding complications due to tPA & $0(0 \%)$ \\
\hline
\end{tabular}

$t P A$ tissue plasminogen activator; $P E$ pulmonary embolism; $C T A$ computed tomography angiography

discharged to long term care facility and subacute rehabilitation facility, respectively. Among 51 deceased patients, the causes of death included ARDS and septic shock solely due to COVID-19 in $90.2 \%$ (46/51). 3 patients died of extensive lung fibrosis complicated by multiple pneumothoraces, septic shock and ARDS due to bacteremia, and hemorrhagic shock due to unilateral hemothorax unrelated to tPA infusion, respectively, even after they survived COVID-19 related septic shock and ARDS. As presumed contributing causes of death, 1 patient had fungemia and RV thrombus, and 1 patient had bacteremia, respectively, in addition to COVID-19 related septic shock and ARDS. 


\section{Discussion}

To our knowledge, this is the largest retrospective cohort study describing the clinical characteristics and outcomes of critically ill COVID-19 patients with a presumed PE based on worsening hypoxia and, or hypotension who were treated with tPA. In contrast to an earlier report describing good outcomes in three patients who received tPA for COVID-19 related severe hypoxia, who had no evidence of RV strain or DVT [11], the $89.5 \%$ mortality of our patients who received tPA was significantly higher. In our study, $47.4 \%$ of patients after tPA had initial improvement in either P/F ratio, blood pressure or hypercarbia. This finding suggests that highrisk pulmonary thromboembolism may have been the main contributing factor responsible for the patients worsening clinical status. Thromboembolic complications are highly prevalent in hospitalized critically ill patients admitted with COVID-19. In a recent systematic review and meta-analysis describing the incidence of VTE among hospitalized patients with COVID-19, the overall estimated pooled incidence of VTE was $17 \%$, with a higher rate of $27.9 \%$ in the critically ill [18]. In a study comparing VTE incidence in patients with non-COVID-19 ARDS, who were propensity matched for equivalent severity of illness with patients with COVID-19 ARDS, the incidence of VTE in patients with COVID-19 was significantly higher [19].

We were not able to confirm the diagnosis of acute PE by Chest CTA as our patients were too unstable for transportation to radiology department. Of note, using bedside point of care ultrasound (POCUS), RV strain was detected in 33.3\% of patients, all of whom had RV dilation with or without septal motion abnormality. Although demonstrating RV thrombus or new findings of RV strain in hemodynamically unstable hypoxic patients may help to support the emergency use of thrombolytic therapy for a presumed PE [20], RV strain can also be explained by pulmonary hypertension due to hypoxic pulmonary vasoconstriction in the setting of prolonged period of hypoxia in our critically ill COVID19 patients with COVID-19 pneumonia. We used the presence of Vd to support evidence of PE in 43.9\% (25/57) of patients, as manifest by hypercapnia and high minute ventilation. Normally, hypercapnia from decreased capillary perfusion and $\mathrm{Vd}$ from a significant PE results in an increase in minute ventilation resulting in a normal or low arterial $\mathrm{PaCO} 2$ [21]. However, hypercapnia can occur in association with severe pulmonary emboli [22]. Mauri et al., in a small prospective physiologic study of ARDS in COVID-19 patients, elevated dead space fraction was demonstrated to be a specific pathophysiology finding [23], relating to PE as a cause of rapid deterioration in COVID-19 patients.

Although we were able to identify 43 patients who had clinical and physiological abnormalities supporting a diagnosis of PE, the rest of all 14 patients had severe hypoxia and hypotension that led providers to consider PE as the cause of deterioration but had neither documented evidence of RV abnormalities, DVT, RV thrombus nor hypercapnia. The overall $89.5 \%$ mortality of our cohort treated with tPA for presumed PE should first be interpreted in the context of prior literature describing mortality in non-COVID-19 patients receiving tPA, where the risk of dying is approximately $70 \%$ if cardiopulmonary arrest occurs, and mortality is at least $30 \%$ if there is shock requiring inotropic support [24-26]. The study patients who were all presumed to have a significant or high-risk PE, also had severe hypoxemia from ARDS secondary to COVID-19. The described mortality rate, for patients with COVID-19 hypoxic respiratory failure who require mechanical ventilation, independent of a concurrently diagnosed PE, has been described as over $75 \%$, if patients are older and have comorbid conditions [27-29]. The mean age of the study cohort was 60 years, and there was a high prevalence of comorbid conditions including hypertension, obesity, coronary artery disease and diabetes which have all been independently associated with worse outcome in patients hospitalized with COVID-19 [30, 31]. It should be noted that $77.2 \%$ of the study patients received steroids which have been associated with a reduction in mortality in patients receiving mechanical ventilation for COVID-19 respiratory failure [32].

On analysis of the 6 survivors, 2 patients initially required higher level of organ support with ECMO, and 4 patients had immediate clinical improvement following tPA infusion. Considering the rapid deterioration of these patients at the time of tPA, these patients may not have survived without tPA.

As of February 28, 2021, the American Society of Hematology (ASH) updated their recommendations on the use of chemical prophylaxis in hospitalized patients with COVID19 pneumonia, discouraging the empiric use of therapeuticintensity UFH or LMWH for patients requiring ICU level of care in the absence of an otherwise firm indication for therapeutic anticoagulation. This recommendation was made after an interim pooled analysis in 3 multiplatform trials (REMAP-CAP, ATTACC and ACTIV-4A) demonstrated both the futility of empirical use of therapeutic anticoagulation to reduce the need for organ support over the first 21 days of hospitalization, and the significant rate of bleeding in patients studied [33]. In contrast, for hospitalized patients not requiring ICU level of care, the National Institutes of Health recommended on January 22, 2021 that therapeutic dose anticoagulation is superior to usual prophylactic dose anticoagulation in reducing the need for organ support and mortality [34]. ASH encourages participation in ongoing clinical trials. In our study, tPA was given to $77.2 \%$ patients for a presumed high-risk PE who were already 
receiving therapeutic anticoagulation. The current recommendations from ASH may result in the decrease both of empiric use of therapeutic dose anticoagulation for critically ill COVID-19 patients and the use of tPA for a presumed PE. A recent position paper from The National Pulmonary Embolism Response Team (PERT) Consortium recommends the administration of systemic thrombolytic therapy who have imminent or ongoing hemodynamic collapse, and a presumed PE, even in the absence of a diagnostic Chest CTA, doppler ultrasound of the extremities, or a transthoracic echocardiogram demonstrating $\mathrm{RV}$ dysfunction. While systemic thrombolysis is recommended for patients without COVID-19 and high-risk PE [35], the decision to use systemic thrombolysis for critically ill COVID-19 patients without confirmed PE should be made after appropriate consultation with an in-hospital PERT, if available [36].

There are certain limitations to our study including its retrospective design, and the inclusion of a relatively small cohort of patients. We describe patients who received tPA for a presumed PE, not a documented PE. All the patients described were too unstable for transportation to receive a Chest CT Angiogram. There was no control group, and we did not evaluate outcomes in patients with critically ill COVID-19 and suspected PE who did not receive tPA. The study represents patients only from the New York metropolitan area, however the patients described are from a large and diverse population. We rely on data from an electronic health record data base and therefore we may be missing significant information.

\section{Conclusions}

In our cohort of critically ill mechanically ventilated COVID-19 patients with acute hypoxic respiratory failure treated with tPA for a presumed PE, for worsening hypoxemia and, or hemodynamic instability, the mortality rate was $89.5 \%$. This is despite $47.4 \%$ of patients demonstrating initial improvements in either $\mathrm{P} / \mathrm{F}$ ratio, blood pressure or hypercarbia following tPa. Further studies are required to examine the utility of tPA for critically ill COVID-19 patients with a high clinical suspicion for PE.

Acknowledgements The authors wish to thank all the clinical staffs who were in care of the patients in Mount Sinai Health System.

\begin{abstract}
Author contributions All the authors assume responsibility for all content of the manuscript. TK and DS contributed significantly to the conceptualization, MS contributed to the collection, analysis and drafting. DS, TK, NE, MT contributed significantly to the final editing of the manuscript. All the authors reviewed the draft and approved the final version of the manuscript.
\end{abstract}

Funding None.
Data availability Original data files are fully available if required to corresponding author.

\section{Declarations}

Conflict of interest None of the authors have conflict of interest.

Ethical approval This study was approved by Institutional Review Board of Ichan School of Medicine Mount Sinai (\#2000495).

\section{References}

1. Lu R, Zhao X, Li J et al (2020) Genomic characterization and epidemiology of 2019 novel coronavirus: implications for virus origins and receptor binding. Lancet 395(10224):565-574

2. Cui S, Chen S, Li X et al (2020) Prevalence of venous thromboembolism in patients with severe novel coronavirus pneumonia. $\mathrm{J}$ Thromb Haemost 18(6):1421-1424

3. Escher R, Breakey N, Lämmle B (2020) Severe COVID-19 infection associated with endothelial activation. Thromb Res 190:62

4. Tang N, Li D, Wang X et al (2020) Abnormal coagulation parameters are associated with poor prognosis in patients with novel coronavirus pneumonia. J Thromb Haemost 18(4):844-847

5. Ackermann M, Verleden SE, Kuehnel M et al (2020) Pulmonary vascular endothelialitis, thrombosis, and angiogenesis in Covid19. N Engl J Med 383:120

6. Klok FA, Kruip MJHA, van der Meer NJM et al (2020) Incidence of thrombotic complications in critically ill ICU patients with COVID-19. Thromb Res 191:145

7. Bilaloglu S, Aphinyanaphongs Y, Jones S et al (2020) Thrombosis in hospitalized patients with COVID-19 in a New York City health system. JAMA 324:799

8. Bikdeli B, Madhavan MV, Jimenez D et al (2020) COVID-19 and thrombotic or thromboembolic disease: implications for prevention, antithrombotic therapy, and follow-up: JACC state-of-the-art review. J Am Coll Cardiol 75(23):2950-2973

9. Connors JM, Levy JH (2020) COVID-19 and its implications for thrombosis and anticoagulation. Blood 135(23):2033-2040

10. Goyal A, Saigal S, Niwariya Y et al (2020) Successful use of tPA for thrombolysis in COVID related ARDS: a case series. J Thromb Thrombolysis 2:1-4

11. Poor HD, Ventetuolo CE, Tolbert $\mathrm{T}$ et al (2020) COVID-19 critical illness pathophysiology driven by diffuse pulmonary thrombi and pulmonary endothelial dysfunction responsive to thrombolysis. Clin Transl Med 10(2):e44

12. Ghia S, Bhatt H, Lazar M (2020) Role of tissue plasminogen activator for diffuse pulmonary microemboli in coronavirus disease 2019 patient. J Cardiothorac Vasc Anesth S1053-0770(20):30858-30862

13. Rivera-Lebron B, McDaniel M, Ahrar K, PERT Consortium et al (2019) Diagnosis, treatment and follow up of acute pulmonary embolism: consensus practice from the PERT Consortium. Clin Appl Thromb Hemost. 25:1076029619853037

14. Le Gal G, Righini M, Sanchez O et al (2006) A positive compression ultrasonography of the lower limb veins is highly predictive of pulmonary embolism on computed tomography in suspected patients. Thromb Haemost 95:963-966

15. Labovitz AJ, Noble VE, Bierig M et al (2010) Focused cardiac ultrasound in the emergent setting: a consensus statement of the American Society of Echocardiography and American College of Emergency Physicians. J Am Soc Echocardiogr 23(12):1225-1230 
16. Elliott CG (1992) Pulmonary physiology during pulmonary embolism. Chest 101(4 Suppl):163S-171S

17. Schulman S, Kearon C, Subcommittee on Control of Anticoagulation of the Scientific and Standardization Committee of the International Society on Thrombosis and Haemostasis (2005) Definition of major bleeding in clinical investigations of antihemostatic medicinal products in non-surgical patients. J Thromb Haemost 3(4):692-694

18. Jiménez D, García-Sanchez A, Rali P et al (2020) Incidence of VTE and bleeding among hospitalized patients with coronavirus disease 2019: a systematic review and meta-analysis. Chest S0012-3692(20):35146-35151

19. Helms J, Tacquard C, Severac F, CRICS TRIGGERSEP Group (Clinical Research in Intensive Care and Sepsis Trial Group for Global Evaluation and Research in Sepsis) et al (2020) High risk of thrombosis in patients with severe SARS-CoV-2 infection: a multicenter prospective cohort study. Intensive Care Med 46(6):1089-1098

20. Fields JM, Davis J, Girson L et al (2017) Transthoracic echocardiography for diagnosing pulmonary embolism: a systematic review and meta-analysis. J Am Soc Echocardiogr 30(7):714-723.e4

21. Stoelting RK, Dierdorf SF (2002) Anesthesia and co-existing disease, 4th edn. Churchill Livingstone, New York

22. Van Marcke C, Daoudia A, Penaloza A et al (2015) CO2 measurement for the early differential diagnosis of pulmonary embolismrelated shock at the emergency department: a case series. Respir Med Case Rep 11(16):106-108

23. Mauri T, Spinelli E, Scotti E et al (2020) Potential for lung recruitment and ventilation-perfusion mismatch in patients with the acute respiratory distress syndrome from coronavirus disease 2019. Crit Care Med 48(8):1129-1134

24. Jaff MR, McMurtry MS, Archer SL, American Heart Association Council on Cardiopulmonary, Critical Care, Perioperative and Resuscitation, American Heart Association Council on Peripheral Vascular Disease, American Heart Association Council on Arteriosclerosis, Thrombosis and Vascular Biology et al (2011) Management of massive and submassive pulmonary embolism, iliofemoral deep vein thrombosis, and chronic thromboembolic pulmonary hypertension: a scientific statement from the American Heart Association. Circulation 123(16):1788-830

25. Goldhaber SZ, Visani L, De Rosa M (1999) Acute pulmonary embolism: clinical outcomes in the International Cooperative Pulmonary Embolism Registry (ICOPER). Lancet 353(9162):1386-1389

26. Kucher N, Rossi E, De Rosa M et al (2006) Massive pulmonary embolism. Circulation 113(4):577-582

27. Richardson S, Hirsch JS, Narasimhan M et al (2020) Presenting characteristics, comorbidities, and outcomes among 5700 patients hospitalized with COVID-19 in the New York City area. JAMA 323(20):2052-2059

28. Yang X, Yu Y, Xu J et al (2020) Clinical course and outcomes of critically ill patients with SARS-CoV-2 pneumonia in Wuhan, China: a single-centered, retrospective, observational study. Lancet Respir Med 8(5):475-481

29. Guan WJ, Liang WH, Zhao Y, China Medical Treatment Expert Group for COVID-19 et al (2020) Comorbidity and its impact on 1590 patients with COVID-19 in China: a nationwide analysis. Eur Respir J 55(5):2000547

30. Bhatraju PK, Ghassemieh BJ, Nichols M et al (2020) Covid-19 in critically Ill patients in the Seattle region-case series. N Engl J Med 382(21):2012-2022

31. Grasselli G, Zangrillo A, Zanella A, COVID-19 Lombardy ICU Network et al (2020) Baseline characteristics and outcomes of 1591 patients infected with SARS-CoV-2 admitted to ICUs of the Lombardy region, Italy. JAMA 323(16):1574-1581

32. RECOVERY Collaborative Group, Horby P, Lim WS, Emberson JR et al (2021) Dexamethasone in hospitalized patients with Covid-19. N Engl J Med 384(8):693-704

33. Lee A, deSancho M, Pai M, et al (2020) COVID-19 and pulmonary embolism: frequently asked questions. American Society of Hematology. COVID-19 Resources Web site. Version 8.0 updated January 29, 2021. Available from https://www.hematology.org/ covid-19/covid-19-and-vte-anticoagulation. Accessed 28 Feb 2021

34. National Heart, Lung and Blood Institute (2021) ATTACC, ACTIV-4a \& REM-CAP multiplatform RCT results of interim analysis. Retrieved from https://nhlbi-connects.org/documents/ mpRCT\%20Interim\%20Presentation.pdf. Accessed 22 Mar 2021

35. Kearon C, Akl EA, Comerota AJ et al (2012) Antithrombotic therapy for VTE disease: antithrombotic therapy and prevention of thrombosis, 9th ed: American College of Chest Physicians evidence-based clinical practice guidelines. Chest $141(2$ Suppl):e419S-e496S

36. Rosovsky RP, Grodzin C, Channick R et al (2020) Diagnosis and treatment of pulmonary embolism during the coronavirus disease 2019 pandemic: a position paper from the National PERT Consortium. Chest 158(6):2590-2601

Publisher's Note Springer Nature remains neutral with regard to jurisdictional claims in published maps and institutional affiliations. 